\title{
UN ANÁLISIS DE LAS PROPIEDADES COMBINATORIAS DE LOS PRIMITIVOS SEMÁNTICOS A TRAVÉS DE LAS ADPOSICIONES COMPLEJAS EN INGLÉS ANTIGUO*
}

\author{
María del Carmen Guarddon Anelo
}

UNED

\section{RESUMEN}

Este trabajo constituye un estudio del contenido léxico de una serie de adposisiciones complejas en inglés antiguo y los procesos morfo-semánticos que las originaron. Concretamente, he analizado las adposiciones que tienen como elementos controladores in, on y at. El marco teórico utilizado es el Metalenguaje Semántico Natural (en adelante, MSN). Mi objetivo principal es demostrar que la explicación de las propiedades combinatorias de los primitivos espaciales propuestos en el MSN puede servir para aclarar aspectos de la gramática del léxico espacial que no han sido tratados todavía por escuelas altamente centradas en esta cuestión, como la Lingüística Cognitiva.

Los primitivos espaciales se usan aquí para dilucidar tres cuestiones fundamentales: 1) Contenido semántico de la adposición resultante: significados de los constituyentes heredados por el compuesto, significados que quedan bloqueados en el proceso y nuevos significados que emergen en el compuesto; 2) Las incompatibilidades semánticas que evitan la combinación de algunas adposiciones y 3) La organización sintáctica interna que encontramos en estas adposiciones. 4) La evolución diacrónica de las adposiciones complejas analizadas. Propongo una serie de factores semánticos como origen de la desaparición de algunas de estas adposiciones mientras que, en cambio, otras han llegado hasta nuestros días.

Palabras clave: Lenguaje Semántico Natural, adposiciones complejas, propiedades combinatorias de los primitivos espaciales, diacronía del léxico espacial.

\section{ABSTRACT}

This paper presents a study of the lexical content of a number of complex adpositions in Old English and the morpho-semantic processes that have motivated them. Specifically, I have analysed the adpositions which have in, on and at as controlling elements. The theoretical framework supporting this analysis is the Natural Semantic Metalanguage (henceforth, NSM). The main goal of this paper is to demonstrate that the explication of the combinatorial properties of the spatial primes put forward in the NSM can serve to shed light upon certain aspects of the grammar of spatial relations that have not received attention by schools highly focused in this type of metalanguage, i.e. Cognitive Linguistics.

\footnotetext{
* Este trabajo ha sido financiado por el proyecto HUM2005-07651-Co202/FILO.
} 
The spatial primes are used to address four fundamental issues: 1) Semantic content of the resulting adposition; senses of the constituent elements that are transferred to the complex adposition, senses that are blocked and senses not present in the constituents that arise in the process of compounding; 2) Semantic incompatibilities preventing the combination of certain simple adpositions; 3) Internal syntactic organization found in these complex adpositions. 4) The diachronic evolution of the complex adpositions analyzed in the article. Particularly, I unveil the semantic factors that have led to the disappearance of some of these adpositions while others have survived up to the present day.

Key Words: Natural Semantic Metalanguage, complex adpositions, combinatorial properties of the semantic primes, diachronic evolution of the spatial lexicon.

\section{INTRODUCCIÓN}

Las últimas décadas han sido testigo de una producción ingente de trabajos de semántica sobre adposiciones, más concretamente preposiciones, dentro del marco de la Lingüística Cognitiva. La relevancia de estos elementos dentro de esta escuela se basa en la hipótesis de que el espacio juega un papel determinante en la formación de conceptos más abstractos y más difícilmente comprensibles para la razón humana. En cualquier caso, esta extensa literatura apenas ha alcanzado consenso, primero, en la metodología que debería emplearse para describir el significado de las adposiciones, y segundo, y tal vez como una consecuencia de lo anterior, en cuanto a si su contenido semántico es reductible a un único significado general, o, por el contrario son elementos altamente polisémicos. Además, está la cuestión fundamental de la universalidad. La Lingüística Cognitiva propone la existencia de procesos de conceptualización que surgen de la interacción de nuestro cuerpo con el entorno. Como tales, se suponen que son universales y esta universalidad debería reflejarse en el lenguaje, independientemente de la idiosincrasia de cada lexicón y de cada gramática. La gran mayoría de estos análisis se han aplicado a una lengua, el inglés, y a un período específico de esta lengua, el inglés actual.

Hasta este momento, las representaciones diagramáticas y proposicionales han competido para llegar a una descripción de las adposiciones inglesas. De todos modos, a pesar de la gran cantidad de métodos desarrollados, y a pesar de que algunos han logrado en gran medida los objetivos de los correspondientes autores, ninguno de ellos ha resultado ser idóneos para la descripción del contenido léxico de las adposiciones.

En este trabajo analizo las adposiciones complejas controladas por in, on y at con el objeto de explicar los procesos composicionales que las han producido y sus tendencias evolutivas. El marco teórico utilizado para elaborar este trabajo es el Metalenguaje Semántico Natural (en 
adelante, MSN) propuesto por Goddard y Wierzbicka 2002 y Wierzbicka 2002. La herramienta de análisis fundamental empleada son una serie de primitivos semánticos, que constituyen la piedra angular del MSN.

En la Sección 2, explico con más detalle de qué manera estos primitivos son relevantes en el estudio de los procesos composicionales que implican a las adposiciones en inglés antiguo y a la comprensión de los cambios que los datos han experimentado, cuando son objeto de contraste con la situación actual de la lengua (Martín Arista 2005; de la Cruz 2006). Asimismo, en esta misma sección introduzco el inventario de datos que han sido analizados. En la Sección 3, describo el significado de las adposiciones analizadas utilizando los primitivos semánticos relevantes. En la Sección 4, a partir de una observación de las tendencias de combinación de estos primitivos, extrapolo reglas que definen las propiedades combinatorias de las adposiciones inglesas in, on y at a nivel semántico y morfo-sintáctico (Martín Arista, en prensa-b). Debido a que la mayor parte de las adposiciones complejas han caído en desuso, también intento establecer principios que predigan su desaparición en estadios posteriores de la lengua. Si la evolución de un número notable de los elementos estudiados puede ser explicado por las mismas reglas composicionales, cuento con una razón de peso para considerar que estos principios caracterizan significativamente el comportamiento diacrónico de las adposiciones locativas en inglés. La eficacia de estos principios se basa en su capacidad para aclarar problemas arduos como las restricciones en la integración de los elementos léxicos en una unidad compleja y su repercusión en una teoría del cambio semántico del léxico espacial en inglés.

\section{Las Adposiciones Complejas en Inglés Antiguo y el EnfoQue del Metalenguaje Semántico Natural}

Las adposiciones in, on y at en inglés antiguo aparecen como palabras independientes y en composición con otras adposiciones. También pueden aparecer como fonemas trabados o prefijadas a diferentes partes de la oración. No obstante, en este trabajo me voy a centrar solamente en los casos en los que, junto a otras adposiciones, generan adposiciones complejas. Además, incluiré en los datos examinados las adposiciones complejas onemn 'cerca de', ethindan 'detrás de', y ongemong 'entre', las cuales están compuestas de una adposición y un adjetivo, un adverbio y un sustantivo respectivamente. La elección de in, on y at, se justifica por el hecho de que precisamente estas adposiciones muestran una tendencia evidente a participar en procesos de formación de palabras con miembros de la misma categoría. Existe un número muy elevado de adposiciones complejas que cuentan con una de estas adpo- 
siciones como su primer elemento, mientras que los casos de adposiciones complejas donde otros elementos aparecen en primer lugar es con diferencia menor, por ejemplo betweon 'entre dos'. Por otro lado, se dan muy pocos casos donde in, on y at aparezcan en segunda posición, tales como upon 'sobre' o within 'dentro'. Sólo incluiré en el análisis las adposiciones complejas donde in, on y at aparecen en posición inicial, ya que tomo como premisa que la configuración sintáctica interna de las adposiciones tiene consecuencias en el contenido semántico resultante.

Finalmente, debo hacer notar que parte de los elementos léxicos examinados en este artículo también funcionan como otras categorías que no son adposiciones, por ejemplo, adverbios. En cualquier caso, el significado de estas partes de la oración también será incluido en el análisis, por si pueden arrojar algo de luz sobre los objetivos fundamentales de este trabajo.

Con objeto de enumerar las adposiciones analizadas es más instructivo organizarlas en una presentación tabular, como muestro a continuación (Tabla 2.1). En esta tabla, las adposiciones complejas en inglés antiguo están organizadas en tres grupos según la adposición que se emplaza en posición inicial, bien in, on o at. Los significados de cada adposición y la referencia a las diferentes categorías funcionales han sido extraídos y traducidos al español de A Concise Anglo-Saxon Dictionary (Clark Hall 1894/1960). Esta no es la única forma posible de agrupar estas adposiciones pero contribuye a clarificar la exposición de los datos, como acabo de apuntar.

La cuestión analítica fundamental que subyace a todo este trabajo es si existen tendencias fijas en las propiedades combinatorias de estas adposiciones. De esta cuestión se derivan las cuatro que constituyen el eje de este artículo y que he avanzado en el resumen:

1. ¿Cómo se deriva el significado de la adposición compleja a partir del de sus constituyentes?

2. ¿Es posible extraer reglas combinatorias que operan en el proceso de composición? En otras palabras, ¿puede uno aventurarse a afirmar que existen elementos que nunca aparecerían combinados en una adposición compleja?

3. ¿Qué patrones sintácticos siguen estos elementos? ¿Es uno de los elementos constituyentes el dominante desde el punto de vista morfosintáctico y/o semántico?

4. ¿Cómo determinan los patrones combinatorios la evolución diacrónica de estas adposiciones?

Concretamente, trataríamos de explicar la desaparición de algunas de ellas frente a la supervivencia de otras hasta nuestros días. 
TABLA 2.1: Las adposiciones in, on y at y los compuestos en los que aparecen.

\begin{tabular}{|c|c|c|}
\hline$I_{N}$ & CATEGORÍAS ASOCIADAS & SIGNIFICADO \\
\hline \multirow[t]{2}{*}{ In } & Preposición & $\begin{array}{l}\text { en, dentro, sobre, hacia, a, entre, } \\
\text { alrededor, durante }\end{array}$ \\
\hline & Adverbio & en, hacia dentro, dentro \\
\hline \multirow[t]{2}{*}{ Inn-an } & Preposición & en, entre, en el interior de \\
\hline & Adverbio & dentro de \\
\hline$O_{N}$ & CATEGORÍAS ASOCIADAS & SIGNIFICADO \\
\hline On & Preposición & $\begin{array}{l}\text { sobre, encima de, hasta, entre, } \\
\text { en, hacia dentro, contra, hacia, } \\
\text { según, de acuerdo a, con respecto a, } \\
\text { por, a cambio de }\end{array}$ \\
\hline \multirow[t]{2}{*}{ On-butan } & Preposición & encima de \\
\hline & Adverbio & sobre, alrededor \\
\hline \multirow[t]{2}{*}{ On-emn } & Preposición & cerca de, durante \\
\hline & Adverbio & juntamente, exactamente, directamente \\
\hline \multirow[t]{2}{*}{ On-foran } & Preposición & antes de, al comienzo de \\
\hline & Adverbio & antes, delante de \\
\hline \multirow[t]{2}{*}{ On-gean } & Preposición & $\begin{array}{l}\text { contra, en frente de, al contrario de, } \\
\text { a cambio de }\end{array}$ \\
\hline & Adverbio & al otro lado, en la parte de atrás \\
\hline \multirow[t]{2}{*}{ On-gemong } & Preposición & entre \\
\hline & Adverbio & $\begin{array}{l}\text { durante este periodo, mientras, } \\
\text { al mismo tiempo }\end{array}$ \\
\hline On-inn-an & Preposición & dentro de \\
\hline \multirow[t]{2}{*}{ On-ufan } & Preposición & encima de, sobre, más allá de, detrás de \\
\hline & Adverbio & encima, sobre, más allá \\
\hline \multirow[t]{2}{*}{ On-uppan } & Preposición & encima de, sobre \\
\hline & Adverbio & además, encima de eso \\
\hline$A T$ & CATEGORÍAS ASOCIADAS & SIGNIFICADO \\
\hline At & Preposición & $\begin{array}{l}\text { en, a, cerca de, por, dentro de, encima de, } \\
\text { sobre, con, antes de, al lado de, } \\
\text { en cuanto a, hasta, dentro de, hacia, } \\
\text { a la hora de, cerca de, a través de, } \\
\text { desde, por }\end{array}$ \\
\hline \multirow[t]{2}{*}{ Et-foran } & Preposición & $\begin{array}{l}\text { ante, en presencia de, delante de, } \\
\text { cerca de, por }\end{array}$ \\
\hline & Adverbio & de antemano \\
\hline Et-hindan & Preposición & detrás de, después de \\
\hline
\end{tabular}


Repasando la Tabla 2.1, se puede ver que a la mayor parte de estas adposiciones complejas se les ha adscrito un gran número de significados distintos y, en ocasiones, no evidentemente relacionados. En efecto, el significado de estos elementos es muy sensible a dinámicas contextuales y el lector tiene que seleccionar un significado a partir de un uso concreto. Esto evoca un problema contumaz en el estudio de las adposiciones; siendo elementos dotados de flexibilidad semántica son capaces de «negociar» sus significados con un número casi infinito de contextos. Ello ha impulsado a los lingüistas a intentar perfilar un significado central, o un número de significados lo más definido posible que pueda ordenar toda la variación contextual a la que están sujetas las adposiciones. Esta situación da lugar a un obstáculo difícilmente soslayable: ¿Qué significado de cada uno de los componentes polisémicos de estas adposiciones posee un rol activo en el proceso composicional que conduce al contenido semántico del elemento léxico resultante y qué criterios deberían utilizarse para identificar dicho significado? A su polisemia intrínseca tenemos que sumarle su polisemia funcional; pueden funcionar como diversas partes de la oración como muestra la misma tabla.

La Lingüística Cognitiva ofrece una potencial solución a este problema inicial en la forma de los prototipos. Diversos estudios revelan que el significado prototípico es el primero que los hablantes son capaces de evocar al ser confrontados con una palabra dada. Esto puede motivarnos a concluir que es este significado también el más dinámico a la hora de producir nuevo léxico. Sin embargo, establecer el prototipo de estas unidades semánticas no necesariamente tiene que aportar resultados fiables por diversos motivos. Primero, porque por razones obvias no hay hablantes vivos de inglés antiguo que nos pudieran ayudar con su intuición. En segundo lugar, porque los ejemplos de estas categorías con los que contamos se limitan a unos cuantos textos que han llegado hasta nuestros días, lo que nos obliga a establecer el prototipo a partir de un muestreo muy reducido. En otras palabras, no podríamos utilizar tampoco la frecuencia como un parámetro indicativo de prototipicalidad. A la escasez de textos hay que sumarle la relativa baja frecuencia de la mayoría de las adposiciones complejas. Tampoco podemos basarnos en el conocimiento que tenemos de su uso en inglés actual ya que gran parte de estas adposiciones ha desaparecido.

Por todo lo anteriormente expuesto, el Metalenguaje Semántico Natural se revela como una herramienta muy conveniente a la hora de proporcionar soluciones a estos problemas. Como se apuntó brevemente antes, esta teoría propone la existencia de unidades léxicas universales, «primitivos», que son indefinibles y que se encuentran en todas las lenguas, lo que nos permite calificarlas como universales. Como su nombre evidencia, los primitivos no pueden reducirse en ningún grado o para- 
frasearse a través de otros elementos léxicos. La substanciación morfológica de los primitivos es variable. Por ejemplo, uno de los primitivos identificados por Goddard y Wierzbicka 2002 es TIEMPO. En inglés este primitivo se codifica a través de un sustantivo, pero esta forma no es extrapolable a todas las lenguas existentes, pues podría adoptar cualquier otra estructura, como la de un sintagma o un morfema ligado. Resumiendo, un primitivo semántico no se corresponde necesariamente con una forma léxica única.

La ventaja de utilizar el programa del MSN en el estudio de elementos léxicos considerablemente polisémicos como las adposiciones se ha puesto de manifiesto recientemente en el análisis realizado por Goddard 2002 de la preposición on. Concretamente, en su análisis de las adposiciones, este marco de investigación semántica permite delinear dónde se encuentra la frontera entre lo que es intrínseco al significado de la adposición y lo que es «accidental» o una contingencia contextual.

Es relevante señalar que la reducción del contenido semántico de estas adposiciones en términos de primitivos semánticos presenta la ventaja de que, a pesar de ser significados invariables, son compatibles con todos sus ejemplos de uso. Por lo tanto, un análisis en términos de primitivos semánticos contribuye a evitar el peliagudo problema que acabamos de enunciar: la extensa polifuncionalidad de las adposiciones así como su carácter eminentemente polisémico.

Como indiqué previamente, dentro del marco de la Lingüística Cognitiva ha surgido una caudalosa corriente de trabajos sobre preposiciones. En la mayor parte de estos trabajos, y a pesar de apoyarse en diferentes criterios, los estudiosos proponen la existencia de un sentido central o prototípico, o de un significado esquemático en el sentido de Langacker 1987 que subyace a todos los usos de esa preposición. Goddard 2002 ha señalado que gran parte de estas formulaciones presenta dos inconvenientes. En primer lugar, suenan extrañas al hablante de la lengua y en segundo lugar, incluyen nociones que no cuentan con equivalentes en otras lenguas que no sea el inglés, por lo que no son demasiado operativas para análisis contrastivos. Esto constituye un problema, particularmente en el campo de las relaciones espaciales, que es uno de los dominios semánticos más universales.

Adoptar los primitivos del MSN para mi estudio tiene una consecuencia inmediata en relación con las cuatro cuestiones planteadas anteriormente y que me dispongo a dilucidar en este trabajo: el análisis conlleva explorar los patrones combinatorios de los primitivos semánticos espaciales presentes en las adposiciones complejas. En otras palabras, este análisis revelará regularidades estructurales que los primitivos semánticos espaciales presentan al interactuar con otros primitivos semánticos y el tipo de relaciones que son capaces de generar a través de distintos modelos de interacción, en las nuevas adposiciones complejas. 
Aunque no todos los lingüistas suscribirían la perspectiva localista, parece haber un acuerdo a la hora de aceptar que prácticamente la totalidad de las adposiciones originalmente codificó relaciones espaciales. Por ello, con objeto de implementar el análisis de los datos presentados anteriormente, utilizaré los primitivos espaciales identificados por Goddard y Wierzbicka 2002, p. 14 que enumero a continuación, traducidos al español:

Espacio: DONDE/LUGAR, AQUí, ARRIBA, ABAJO, LEJOS, CERCA, LATERAL, DENTRO

A esta compilación yo añadiría el primitivo semántico CONTACTO, propuesto por Goddard 2002 en su análisis de la preposición on que he mencionado anteriormente.

Considerando la primacía del concepto tiempo y la extendida tendencia, atestiguada en un conjunto diverso de lenguas, a expresar relaciones temporales en términos de nociones espaciales, también utilizaré primitivos temporales cada vez que resulte conveniente para la implementación del análisis (Goddard y Wierzbicka 2002 p. 14):

Tiempo: CUANDO/TIEMPO, AHORA, ANTES, DESPUÉS, UN PERÍODO LARGO, UN PERÍODO BREVE, DURANTE

Finalmente, he recurrido a dos primitivos que no expresan nociones espaciales ni temporales, pero que aparecen ejemplificados en algunos de los significados de estas adposiciones. Me refiero al primitivo MOVIMIENTO y al primitivo de similitud COMO. No obstante, debo reconocer que en ocasiones la adscripción de un cierto primitivo semántico a un término se convierte en una cuestión complicada, ya que no siempre la asociación entre un primitivo y su exponente es tan obvia como en el caso de algunos elementos locales. Una de las causas de esta situación es que el conjunto de primitivos semánticos que presenta el modelo es limitado. De otro modo, perdería su valor explicativo y el carácter atómico que en principio caracteriza a estos primitivos. Esto me ha conducido a tomar decisiones delicadas en cuanto al primitivo expuesto por un determinado significado. Particularmente complejo ha sido determinar el primitivo que subyace a la categoría gean cuando funciona como adjetivo y significa «directo». Entre el primitivo determinante THE SAME y el de similitud сомо, me he decantado por el último, porque refleja en mayor medida el valor predicativo de este adjetivo.

En otro orden de cosas, el valor explicativo de los exponentes de los primitivos semánticos está basado en su universalidad, que se ha comprobado en diferentes lenguas. Por esta razón, extrapolando esta característica de permanencia a un análisis diacrónico, se puede encontrar evidencia reveladora de la continuidad de la presencia de estos primitivos en estas preposiciones. Además, analizando los datos de este 
período, creo que es posible establecer reglas predictivas que anticipan la situación de estos elementos léxicos en inglés moderno (ver Martín Arista, en prensa-a).

La Tabla 2.1 mostraba que las adposiciones in, on y at han mantenido su significado relativamente estables hasta el inglés actual. En realidad estaban sujetas a restricciones de selección diferentes a las que presentan en la actualidad, lo que se plasmaba en una alternancia que no refleja el uso actual de estas adposiciones en todos los casos. En lo que respecta a los otros elementos léxicos constitutivos, podemos hablar de una situación más estable, como indican los datos incluidos en la Tabla 2.2, que muestro a continuación:

TABLA 2.2: Componentes léxicos de las adposiciones estudiadas.

\begin{tabular}{|c|c|c|}
\hline AdPOSICIÓN & CATEGORÍAS ASOCIADAS & SIGNIFICADO \\
\hline \multirow[t]{3}{*}{ Butan } & Preposición & $\begin{array}{l}\text { afuera, fuera de, separado de, } \\
\text { alrededor de, excepto, sin, todo excepto, } \\
\text { además de, a pesar de, sin }\end{array}$ \\
\hline & Conjunción & $\begin{array}{l}\text { excepto, excepto que, pero, } \\
\text { con excepción de }\end{array}$ \\
\hline & Adverbio & porfuera, fuera \\
\hline \multirow[t]{2}{*}{ Efen } & Adjetivo & $\begin{array}{l}\text { plano, llano, igual, semejante, parejo, } \\
\text { armonioso, uniforme, tranquilo, plano }\end{array}$ \\
\hline & Adverbio & igualmente, exactamente, justo como \\
\hline \multirow[t]{2}{*}{ Foran } & Preposición & $\begin{array}{l}\text { antes de, enfrente de, en la presencia de, } \\
\text { en favor de, en preferencia a }\end{array}$ \\
\hline & Adverbio & $\begin{array}{l}\text { antes, en frente, hacia delante, } \\
\text { hacia delante, anteriormente, } \\
\text { previamente }\end{array}$ \\
\hline \multirow[t]{2}{*}{ Gean } & Adverbio & $\begin{array}{l}\text { sin embargo, ahora, todavía, de nuevo, } \\
\text { más allá, además, también, } \\
\text { por otra parte, hasta ahora }\end{array}$ \\
\hline & Adjetivo & directo \\
\hline Hindan & Adverbio & desde atrás, atrás \\
\hline Ufan & Adverbio & desde arriba \\
\hline Uppan & Preposición & $\begin{array}{l}\text { encima de, sobre, contra, al (temporal), } \\
\text { después de }\end{array}$ \\
\hline
\end{tabular}

Fundamentar mi previa afirmación no es complicado. Siendo elementos estructurales, pertenecientes a un inventario cerrado, que expresan relaciones entre dos objetos o situaciones, la probabilidad de 
que estos elementos léxicos experimenten cambios semánticos abruptos es, cuando menos, improbable.

\section{Metodología y ANÁLisis de los dATOS}

Antes de entrar de lleno en el método de análisis, me gustaría hacer algunas puntualizaciones en cuanto al tratamiento de los datos:

1. Como he comentado anteriormente, he utilizado las definiciones efectuadas por Clark Hall 1894/1960. Estas definiciones las he traducido al español y así se presentan en las distintas tablas y, en consecuencia, en las explicaciones pertinentes.

2. Cuando hablo de cada una de las adposiciones, me refiero a todos los significados que ostentan en relación con la parte de la oración (preposición, conjunción, etc.) concreta a la que se asocia cada uno de estos significados. Evito de esta manera recurrir a significados prototípicos y no tener en cuenta otros menos representativos, pero que deben ser objeto de análisis igualmente. Cuando varios significados muestran una clara relación de sinonimia me refiero a todos a través de un solo significado lo suficientemente relevante.

3. La ventaja de utilizar los primitivos semánticos para definir las adposiciones tanto simples como complejas se apoya en una cuestión puramente empírica. Estos primitivos pueden aparecer en una definición si tuviéramos que explicar a un hablante el significado concreto de una adposición dada. En consecuencia, creo que la asociación de los primitivos a las adposiciones contribuyen a clarificar el significado de las mismas. Evidentemente, a través de la exposición del hablante a una serie de contextos que recojan la polisemia de la adposición, es como éste realmente entenderá el significado de dicha adposición. Sin embargo, creo que existen distintos niveles de comprensión de un elemento léxico que exprese relaciones estructurales, y la utilización de los primitivos semánticos constituye una manera efectiva de realizar una definición léxica de categorías tan complejas en cuanto su contenido semántico como las adposiciones.

4. Cuando trato la composición de las distintas adposiciones realizo los cortes morfémicos de manera que reflejen la existencia de elementos léxicos en uso en inglés antiguo; es decir suficientemente transparentes para los hablantes de la época. De esta manera, no realizo el análisis del morfema adverbial -an, presente en casi la totalidad de las adposiciones estudiadas y, por ende, oscuro en cuanto a su aportación a la semántica de las mismas. 
Como se refleja en la Tabla 3.1, que incluyo a continuación, en primer lugar he definido las adposiciones básicas in, on y at en términos de primitivos semánticos. Posteriormente, he dividido las adposiciones complejas en sus elementos constituyentes y he procedido de la misma manera; es decir, he tipificado su significado utilizando la teoría de los primitivos semánticos. Del mismo modo he procedido con las unidades complejas. Uno de los problemas que este análisis revela se asocia al hecho de que las adposiciones constituyen una clase léxica de gran complejidad cuyo significado básico es espacial pero denota relaciones temporales y también abstractas, resultantes de diversos procesos metafóricos. A consecuencia de esto, muy a menudo me he encontrado con elementos léxicos que se corresponden con más de un primitivo semántico, lo que viene a confirmar una vez más la naturaleza polisémica de las adposiciones. La multiplicidad de dominios donde estas adposiciones operan no es la única complicación que encontramos; algunas también presentan significados diferentes en un dominio semántico único, particularmente el espacial. En lo que se refiere a las adposiciones complejas cuyos componentes pertenecen a otras partes de la oración (efen 'incluso', gemong 'gentío', hindan 'detrás', gean 'sin embargo'), he aplicado la misma metodología, puesto que estoy interesada en extraer reglas que restrinjan el proceso de composición que genera términos complejos pertenecientes a la clase cerrada.

Finalmente, cuando uno de los componentes de las adposiciones constituye un caso de polisemia categorial y cada una de las categorías exhibe significados aparentemente no vinculados, solamente el significado y la categoría que claramente participan en el compuesto han sido considerados como relevantes para el análisis. Este es el caso de gean que como adverbio significa 'sin embargo, ahora, todavía, de nuevo, posterior, además, también, por otra parte, y hasta ahora', mientras que como adjetivo significa 'directo', definición que no ostenta una relación evidente con los significados expuestos anteriormente.

La Tabla 3.1 ilustra los primitivos que subyacen a las adposiciones incluidas previamente en la Tabla 2.1. Como apunté anteriormente, da la impresión de que la mayor parte de las adposiciones son al mismo tiempo exponentes de primitivos espaciales y temporales. Esta polisemia se puede explicar en términos de la base experiencial que el concepto de espacio proporciona para la comprensión de las relaciones temporales (Wierzbicka 1993). En cualquier caso, en lugar de considerar esta afirmación como una clave para descifrar los problemas léxicos que puedan aparecer en este sentido, exploraré las consecuencias de esta polisemia a partir de los postulados de la semántica composicional (véase Martín Arista y Martín de la Rosa 2006).

El análisis semántico de cada adposición se presenta seguidamente en el mismo orden en el que aparecen en la Tabla 3.1. 
TABLA 3.1: Primitivos representados por las adposiciones analizadas.

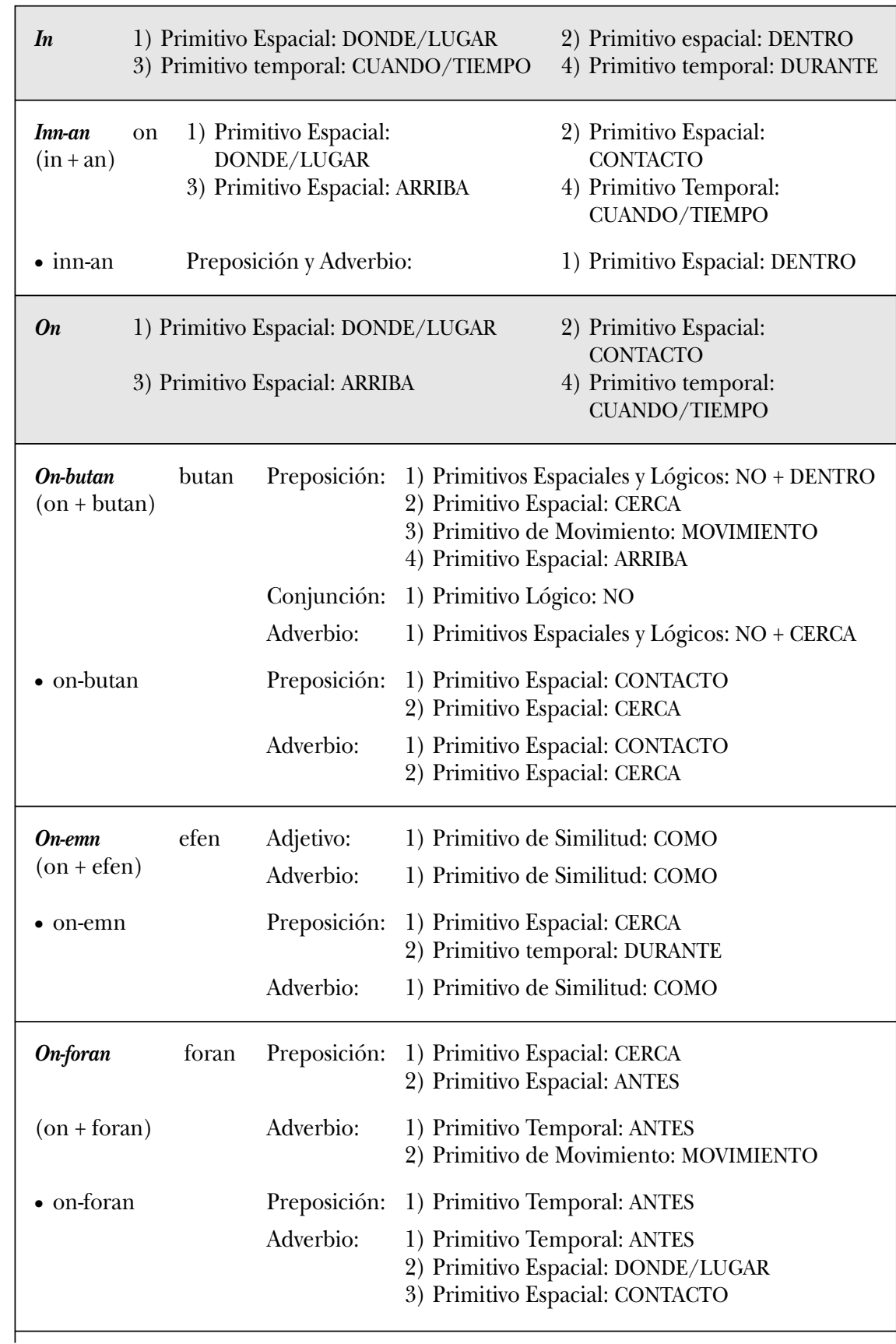




\begin{tabular}{|c|c|c|}
\hline \multirow[t]{2}{*}{$\begin{array}{l}\text { On-gean } \\
(\text { on+gean })\end{array}$} & Adverbio: & $\begin{array}{l}\text { 1) Primitivo Temporal: DESPUÉS } \\
\text { 2) Primitivo Temporal: AHORA }\end{array}$ \\
\hline & Adjetivo: & 1) Primitivo de Similitud: COMO \\
\hline \multirow[t]{2}{*}{ - ongean } & Preposición: & 1) Primitivo Espacial: CONTACTO \\
\hline & Adverbio: & 1) Primitivo Espacial: LATERAL \\
\hline $\begin{array}{l}\text { On-gemong gemong } \\
\text { (on+gemong) }\end{array}$ & Nombre: & 1) Sustantivo: GENTE \\
\hline \multirow[t]{2}{*}{ - ongemong } & Preposición: & 1) Primitivo Espacial: DENTRO \\
\hline & Adverbio: & $\begin{array}{l}\text { 1) Primitivo Temporal: DURANTE } \\
\text { 2) Primitivo Temporal: CUANDO/TIEMPO }\end{array}$ \\
\hline \multicolumn{3}{|l|}{$\begin{array}{l}\text { On-innan } \\
(\text { on }+ \text { in }+ \text { an })\end{array}$} \\
\hline - oninnan & \multicolumn{2}{|c|}{ Preposición: 1) Primitivo Espacial: DENTRO } \\
\hline $\begin{array}{l}\text { On-ufan } \\
(\text { on }+ \text { ufan })\end{array}$ & Adverbio: & 1) Primitivo Espacial: ARRIBA + MOVIMIENTO \\
\hline - on-ufan & Preposición: & $\begin{array}{l}\text { 1) Primitivo Espacial: ARRIBA } \\
\text { 2) Primitivo Temporal: DESPUÉS }\end{array}$ \\
\hline $\begin{array}{l}\text { On-uppan } \\
\text { (on + uppan) }\end{array}$ & Preposición: & $\begin{array}{l}\text { 1) Primitivo Espacial: ARRIBA } \\
\text { 2) Primitivo de Movimiento: MOVIMIENTO } \\
\text { 3) Primitivo Temporal: DESPUÉS + AHORA }\end{array}$ \\
\hline - on-uppan & Preposición: & 1) Primitivo Espacial: ARRIBA \\
\hline 1) Primitivo & Espacial: DOND & $\begin{array}{l}\text { 2) Primitivo Temporal: } \\
\text { CUANDO/TIEMPO }\end{array}$ \\
\hline \multirow[t]{2}{*}{$\begin{array}{l}\text { Et-foran } \\
(\text { Et }+ \text { foran })\end{array}$} & Preposición: & $\begin{array}{l}\text { 1) Primitivo Espacial: CERCA } \\
\text { 2) Primitivo Temporal: ANTES }\end{array}$ \\
\hline & Adverbio: & 1) Primitivo Temporal: ANTES \\
\hline \multirow[t]{2}{*}{ - Et-foran } & Preposición: & $\begin{array}{l}\text { 1) Primitivo Espacial: CERCA } \\
\text { 2) Primitivo Temporal: ANTES }\end{array}$ \\
\hline & Adverbio: & 1) Primitivo Temporal: ANTES \\
\hline $\begin{array}{l}\text { Et-hindan hindan } \\
(\mathbb{E}+\text { hindan })\end{array}$ & Adverbio: & $\begin{array}{l}\text { 1) Primitivo Espacial: DETRÁS } \\
\text { 2) Primitivo de Movimiento: MOVIMIENTO }\end{array}$ \\
\hline - ÆEt-hindan & Preposición: & 1) Primitivo Espacial: DETRÁS \\
\hline
\end{tabular}


1. Innan: Las adposiciones constituyentes in y on son ambas exponentes del primitivo espacial DONDE/LUGAR. Esto indica que existe un cierto grado de sinonimia entre ellas. De hecho, en inglés antiguo su distribución no estaba regulada por las mismas restricciones selectivas que determina su uso en la actualidad. Ambas aparecían en expresiones que ubicaban entidades en regiones, países, e incluso edificios. En Guarddon 2006, se explica que a pesar de la laxitud de este escenario, su uso no era arbitrario, sino que estaba regulado por dinámicas discursivas complejas que afectaban la perspectiva del narrador de la escena y por tanto, la forma en la que ésta era descrita. De todos modos, teniendo en cuenta que este estudio no toma en consideración la dependencia contextual, me ceñiré al contenido léxico de la adposición. Cuando se observa la Tabla 2.1, puede apreciarse que tanto in como on se utilizan para definirse la una a la otra. Esto se debe a que en inglés antiguo, a diferencia de lo que sucede en la actualidad, ambas aparecían en expresiones locativas que no perfilaban configuraciones geométricas específicas como contención dentro de unos límites, o contacto entre dos superficies. A pesar de eso, examinando su uso en inglés antiguo se hace evidente que estas adposiciones, también en esa época, se asociaban a primitivos semánticos diferenciadores. Así, on es exponente del primitivo semántico CONTACTO, mientras que a la preposición in se le adscribe el primitivo semántico DENTRO. Debo subrayar, que el primitivo semántico CONTACTO hace alusión a una relación espacial que expresa la ubicación del trayector ${ }^{1}$ en el hito sin que intervenga una relación de inclusión. Esta relación no tiene que responder a relaciones percibidas visualmente en dos superficies, una del trayector y del hito, pues están en contacto, lo cual se corresponde con el prototipo sincrónico. En lugar de eso, me refiero a relaciones espaciales como las ejemplificadas en volcanic and seismic hazards on the island of Hawaii. Además, ambas adposiciones ejemplifican el primitivo semántico más general DoNDE/LUGAR. La necesidad de establecer un primitivo semántico común para ambas en inglés antiguo es obvia, ya que ambas aparecían en colocación con los mismos hitos. En inglés moderno esto no es tan habitual, pero son varios los casos que podríamos mencionar donde, precediendo al mismo hito, las dos preposiciones expresan ubicación en un lugar, con matices que se derivan precisamente de los primitivos semánticos que las diferencian.

Partimos del supuesto de que estas preposiciones expresan una relación espacial estática. Los significados dinámicos que se les asigna en

\footnotetext{
1 Los términos trayector e hito corresponden a mi traducción de las nociones trajector y landmark introducidas por Langacker 1987. El trayector es el elemento ubicado en una relación predicativa con respecto al hito, que presenta las cualidades típicas de una referencia efectiva (saliencia perceptiva, naturaleza estática con respecto al trayector, etc.). Agradezco a Enrique Bernárdez su propuesta de la traducción de landmark por hito.
} 
algunas definiciones son una consecuencia del contexto lingüístico donde aparecen junto a un verbo de movimiento (Bennet 1975). Por lo tanto, no considero este componente dinámico como parte del contenido léxico de dichas preposiciones, y no sería viable considerarlas exponentes del primitivo MOVIMIENTO. Las dos adposiciones constituyen un claro caso de polisemia, puesto que expresan relaciones en el ámbito temporal, siendo exponentes del primitivo CUANDO/TIEMPO.

Más allá de sus diferencias, estas dos adposiciones comparten un primitivo semántico que se integra en innan. Esta característica ha de anotarse para comprobar si se da sistemáticamente en el resto de los compuestos. La consecuencia más llamativa de su combinación es que una parte fundamental de su naturaleza polisémica se ha perdido: innan no expresa relaciones temporales. En consecuencia, podría decirse que este compuesto ha experimentado una restricción en su dominio de aplicación en comparación con sus constituyentes léxicos. También tendremos en cuenta que este proceso de pérdida de sentidos temporales puede ser recurrente y darse en el resto de las adposiciones.

2. Onbutan: La adposición componente butan es incuestionablemente polisémica. El vínculo entre sus distintos sentidos es la noción espacial: «fuera de». Este concepto no está explícitamente representado en la lista de primitivos del MSN. Por esta razón, esta adposición constituye un caso de portmanteau ya que uno de sus sentidos se explica en función de la combinación de los primitivos NO y DENTRO ${ }^{2}$. No obstante, en aras de la sencillez, para referirme a su significado seguiré utilizando la locución fuera de.

Como preposición, también significa ALREDEDOR, asociada a los primitivos semánticos CERCA y MOVIMIENTO, por lo que estaríamos también ante un caso de portmanteau, en base a la existencia de una relación de movimiento tanto real como de lo que Talmy 1996 denominó «movimiento fictivo». Talmy explicaba con este término la conceptualización de relaciones que, aunque son realmente estáticas, los hablantes las conceptualizan a través de una idea previa de movimiento a la que subyace una trayectoria que también define la relación estática, por ejemplo: hay árboles a lo largo de toda la carretera frente a fue dando tumbos por toda la carretera.

La existencia de un significado que es exponente de CERCA puede justificarse por la relación semántica que existe entre alrededor de y fuera de; para que la ubicación de una entidad con respecto a otra pueda expresarse como fuera de, debe localizarse cerca del objeto de referencia. Esto en principio parece estar lo suficientemente fundamentado.

\footnotetext{
${ }^{2}$ Goddard y Wierzbicka 2002 definen portmanteau como el caso en el que una única palabra expresa una combinación de primitivos semánticos.
} 
Lo complicado es extraer el sentido de movimiento que subyace a uno de los significados de onbutan se trata de «alrededor de». Si recurrimos a representaciones analógicas podemos constatar la existencia de reveladoras semejanzas entre ambas nociones:

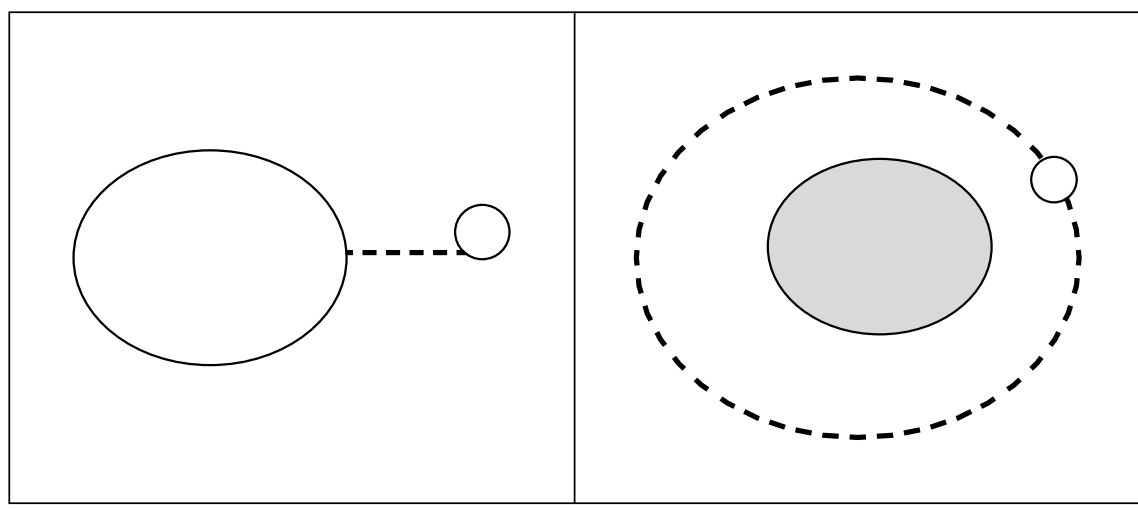

FIGURA 1: Representación de la relación fuera de.

FIGURA 2: Representación de la relación alrededor.

A nivel esquemático en el sentido de Johnson 1987, existe una clara asociación entre ambas representaciones. En primer lugar, la relación fuera de requiere que el trayector esté próximo al hito. Esta proximidad dependerá del tamaño de ambas entidades, de la existencia de una relación funcional entre ellas, de cuestiones de percepción que impliquen a los hablantes, etc. La misma restricción en cuanto a la distancia se da en la relación espacial alrededor de. La única diferencia fundamental es que mientras que en el caso de fuera de el hito tiene la potencialidad de ser un recipiente (por eso lo he representado con un círculo vacío); en el caso de alrededor, la configuración del hito no incluye esa posibilidad (esta razón es la que me ha llevado a representarlo con un círculo opaco, no susceptible de contener al trayector). Veamos con un ejemplo cómo se manifiesta esta asociación: imaginemos a unos individuos sitiados en una cabaña. En un momento dado, pueden describir su situación como están fuera / estamos rodeados. Probablemente, el sentido de «alrededor de» que emergió en primer lugar, fue el que sería estático o ejemplo de movimiento fictivo. En éste intervendrían varios trayectores: hay árboles alrededor de la casa o un único trayector que presente la forma pertinente, hay una alambrada alrededor de la casa. Probablemente, por extensión semántica, y en colocación con verbos de movimiento, como ha sucedido con otras preposiciones, apareció el sentido que encontramos en el perro está correteando alrededor de la casa. 
Finalmente, butan presenta un significado que es definido por el primitivo ARRIBA: 'además de'. Según explican Lakoff y Johnson 1981, el sentido de adición es comprendido espacialmente a través del concepto ARRIBA. Esto ha dado lugar a metáforas conceptuales en inglés como more is up. Esta metáfora es productiva también en nuestra propia lengua, lo que avala al menos un cierto carácter universal vinculado a las nociones incorporadas en las que se basa: como siga acumulando méritos, va a llegar muy alto.

En relación con el significado predicativo del compuesto onbutan, parece que el efecto semántico de on neutraliza el significado negativo de butan, como se muestra en la Tabla 2.1. Por otro lado, como es el caso de innan, onbutan se restringe a la expresión de relaciones espaciales y esto es refrendado por la polisemia categorial del término: tanto cuando funciona como preposición como cuando funciona como adverbio, presenta exclusivamente un significado espacial. La presencia de on en el compuesto parece que refuerza este sentido espacial, mientras que el número de relaciones que butan podía codificar constituía un catálogo destacablemente más amplio.

3. Onemn: Esta preposición compleja no está compuesta de dos adposiciones; el segundo elemento, efen se corresponde con dos partes de la oración: adjetivo y adverbio. Efen no tiene valores espaciales o temporales. Es un exponente del primitivo de similitud cOMO. Por consiguiente, en combinación con on resulta una categoría más complicada de analizar que las anteriores. Como preposición, el compuesto onemn es polisémico, pues expresa relaciones en el dominio espacial y también en el temporal. Uno de sus significados, «cerca de», lo convierte en exponente del primitivo semántico CERCA. La presencia de este significado no es, aparentemente, fácil de explicar si tenemos en cuenta el significado de los elementos constituyentes. Pero una mirada atenta nos permite hallar el principio que explica la emergencia del primitivos semántico CERCA a partir de la combinación de COMO y el valor espacial intrínseco de la adposición on. La noción de similitud y de proximidad espacial son nociones conexas. La similitud puede considerarse como una extensión metafórica de la proximidad espacial: ella llegó a un estado cercano a la locura (es decir: un estado que se parecía a la locura). Parece lógico, por tanto, concluir que la combinación de un elemento que expresa proximidad nocional (similitud) con un elemento léxico espacial, convierte dicha proximidad nocional en proximidad puramente espacial, como la que expresa el primitivo semántico CERCA.

Como adposición temporal, onemn significa 'durante'. Obviamente, el primitivo semántico que define duración temporal es DURANTE. Este significado temporal es coherente con las explicaciones proporcionadas previamente para sus significados espaciales. La duración temporal es- 
tablece identificación entre el desarrollo de una acción y el período de tiempo que ocupa, se produce un solapamiento entre ambos. Por otra parte, no olvidemos que la identificación es un tipo de similitud, lo que es coherente con la presencia del primitivo COMO en la definición de otros sentidos ya explicados.

Todos los significados que acabamos de describir están también relacionados con el significado adverbial de onemn 'exactamente', que de forma clara alude a una identificación entre dos puntos o estadios.

4. Onforan: La adposición componente foran ostenta también, como las anteriores, un alto grado de polisemia. A continuación paso a exponer la interrelación semántica entre sus significados. Están ligados por la noción espacial «delante de». Una propiedad interesante de este concepto es que a pesar de ser espacial, se define por un primitivo temporal: ANTES. Esto es la causa, por ejemplo, de la discrepancia existente entre el significado dominante de la preposición before en inglés actual y su etimología. Originalmente, servía para expresar locación espacial y el significado temporal era una extensión metafórica de ese uso. Por el contrario, hoy en día before es sobre todo utilizada para expresar relaciones temporales. Por esta razón, Wierzbicka 1989 propuso esta palabra, before, para representar el correspondiente primitivo temporal. Esto refleja el sólido vínculo existente entre el tiempo, la locación y el movimiento. Consideremos una fila de objetos cuya cabeza es lo primero que hallamos en nuestra línea de visión. Si avanzamos hacia ellos, percibiremos antes aquéllos que están ubicados más cerca de nosotros y preceden a otros elementos; los que vienen antes son los que están delante. Así se construye la fuerte implicación temporal existente en el uso espacial de before: if somebody runs before me, she will reach her destination earlier (before), «si alguien corre delante de mí alcanzará su destino antes», etcétera.

Resumiendo, el compuesto mantiene el significado básico de foran, pero como ha sucedido con otras adposiciones que he analizado anteriormente, lo hace con algunas restricciones. Principalmente, ha perdido los significados metafóricos tales como «en preferencia a» $\mathrm{O}$ «a favor de». Esta pérdida es manifiesta tanto en el uso preposicional como adverbial de esta palabra. Centrándonos en el uso adverbial, en onforan tampoco encontramos ya el sentido dinámico que presentaba foran (véase Tabla 2.1). Por otro lado, el significado temporal que encontramos en foran se mantiene en el compuesto pero adquiere una dimensión más compleja: como preposición, onforan ofrece el significado «al comienzo de».

5. Ongean: El contenido semántico de este compuesto es altamente complejo. Centrémonos en primer lugar en gean, que funciona como 
adverbio además de como adjetivo. Como adverbio, esta palabra presenta una mesnada de significados que podemos subsumir en los primitivos semánticos DESPUÉS y AHORA. He distinguido dos subgrupos en el conjunto de significados de esta categoría: primeramente, aquél cuyos miembros están relacionados con el primitivo AHORA, con una idea de inmediatez; en segundo lugar, aquél cuyos significados son exponentes de DESPUÉs, que expresa una idea de adición. Como adjetivo, su significado es DIRECTO y le he asignado el primitivo semántico cOMO.

En lo que respecta a ongean, como preposición sus significados pertenecen al campo de lo espacial. Tras examinar la Tabla 2.1, uno puede ver que tiene otros significados metafóricos que parten de los sentidos espaciales básicos contra y en frente de. El significado contra implica movimiento hacia un hito dado, por lo que se puede analizar como un portmanteau que aglutina los primitivos MOVIMIENTO + CONTACTO. El otro significado relevante que esta categoría tiene como preposición es en frente de. Éste es un caso de locación donde el trayector está separado del hito con una orientación específica que convierte la relación de separación en una de oposición física. Cuando funciona como adverbio, transmite un sentido deíctico que toma como referencia el lado opuesto del hito: la parte de atrás y es exponente del portmanteau LEJOS + LATERAL. En pocas palabras, el desarrollo más destacado que ha experimentado el compuesto en relación con los elementos que lo constituyen es la pérdida de los significados temporales expresados por gean.

6. Ongemong: Ongemong es otra adposición compleja que no resulta de la combinación de otras dos adposiciones. Como la Tabla 3.1 muestra, gemong significa 'grupo' o 'multitud'; al no ser una adposición no nos centraremos más en este término. La construcción on gemong 'entre la gente' experimentó un proceso de gramaticalización: la pérdida de contenido léxico generó el significado espacial más general «en medio de», que más tarde adquirió una dimensión temporal, «mientras tanto, al mismo tiempo", que se produjo paralelamente con el desarrollo de dos categorías funcionales: un adverbio y una preposición. Se distingue una clara división entre los contenidos léxicos que esta palabra tiene en sus dos facetas funcionales. Es destacable que como adverbio, el contenido semántico de ongemon exhibe una mayor complejidad que como preposición. Como preposición es exponente de DENTRO. Por otro lado, los dos significados que ongemon ofrece como adverbio asocian a esta palabra con dos primitivos temporales: Uno expresa duración, DURANTE, y el segundo expresa localización temporal, CUANDO/TIEMPO.

7. Oninnan: Los elementos que componen esta preposición compleja ya han sido analizados, en consecuencia, me centraré en el significado del compuesto. El primitivo semántico que subyace a su significado 
es DENTRO, como en el caso de innan. De todos modos, incluso si oninnan e innan tienen significados relacionados, existen diferencias que definen los distintos contextos donde aparecen. Mientras que innan es definido como «en» o «entre» (varios elementos), el significado asignado a oninnan es 'dentro', que corresponde exactamente con la palabra propuesta como el primitivo espacial DENTRO. Hasta cierto punto, puede defenderse que el primer elemento on, aporta a oninnan mayores restricciones de tipo locativo desde el punto de vista de la movilidad y la visibilidad del trayector. Obsérvese que dentro está dotado de una idea de inclusión que no está presente necesariamente en en, y definitivamente no lo está en entre (varios elementos).

8. Onufan: Al analizar el significado de los componentes integrados en esta adposición compleja, es evidente que existe un solapamiento entre ambos. Los dos elementos son exponentes del primitivo semántico ARRIBA. En cualquier caso, debemos enfatizar la diferencia fundamental entre ufan y el compuesto que estamos estudiando, i.e. la presencia del componente dinámico en el primero.

Onufan es un término polisémico. Algunos de sus significados han de ser explicados por otros primitivos aparte de ARRIBA. Estos significados son «más allá»y «después de». Originalmente después de tenía un significado local; en consecuencia, estamos ante un caso que nos recuerda a onforan, analizado anteriormente. Tenemos aquí un primitivo temporal, DESPUÉs, que está siendo utilizado para definir una palabra que tiene un significado local. Esto indica la íntima conexión existente entre la organización del espacio y del tiempo: una entidad que está más allá de otra, está ubicada después de ésta. Menos obvia, sin embargo, resulta la derivación del significado espacial de onufan, que en principio se articula en torno a relaciones de localización canónica sobre un plano horizontal, de la especificidad del significado de ufan determinado deíctivamente: «desde arriba». Se puede proponer como elemento común el de la ubicación del trayector a cierta distancia desde la posición del hablante. Cabría considerar la posibilidad de una extensión metonímica en este sentido.

9. Onuppan: Al estudiar el significado de los integrantes de esta adposición compleja, encontramos que de nuevo se da un considerable solapamiento entre ambos. Los dos elementos son exponentes del primitivo semántico ARRIBA. De todos modos, el compuesto presenta un significado bastante simple en comparación con el que se atribuye a uppan. Esta simplificación se explica, en primer lugar, por la pérdida del componente dinámico expresado por uno de los significados de uppan, 'contra', que constituye un pormanteau que comprende los primitivos CONTACTO + MOVIMIENTO. En segundo lugar, se ha producido en onuppan 
una simplificación en lo que concierne al dominio de significación donde opera, en comparación con uppan. Uppan tiene un significado temporal que no se ha transmitido al compuesto. Estos significados son «al» $\mathrm{y}$ «después de», que substancian los primitivos temporales CuANDO/TIEMPO y DESPUÉS respectivamente.

Esta adposición compleja también funciona como un adverbio. $\mathrm{Su}$ significado, «además», no se halla en ninguno de los elementos componentes, pero se puede derivar metafóricamente del primitivo que define a ambos, ARRIBA. Esta asociación puede sostenerse por el uso de las voz inglesa top 'cima' en la locución on top of that 'para colmo', 'por si fuera poco', 'encima'.

10. Etforan: Al ser esta adposición la primera que vamos a analizar en la que el elemento adscrito a la base es la adposición $e$ t, realizaré algunas puntualizaciones con respecto al significado de la misma. Al igual que en inglés moderno, también en inglés antiguo se utilizaba $a t$ con menor frecuencia que las otras dos adposiciones topológicas. Como en la actualidad, presentaba un contenido léxico más simple que in y on, y no aportaba otras caracterizaciones a la relación locativa más allá de la coincidencia espacial entre el trayector y el hito. Esto se refleja en que su definición se realiza con un único primitivo semántico DONDE/LUGAR. Este tipo de locación se da en casos en los que no existe una percepción detallada del hito o no interesa perfilar dichos detalles. Su simplicidad configuracional es lo que la diferencia de las otras dos; de lo cual se hace eco los primitivos semánticos que se atribuyen a in y on.

Los significados espaciales de etforan son definidos por un primitivo temporal: ANTES. Es exactamente la misma situación que se daba en onforan, por conexiones de significado: un primitivo temporal define un término cuyo significado primario actual es espacial. A pesar del hecho de que ANTES subyazca al significado tanto de foran como del compuesto en el que se integra, existe entre ambos elementos diferencias relevantes que siguen patrones similares a otras adposiciones analizadas previamente. Por ejemplo, en el compuesto se pone de manifiesto una reducción en el número de primitivos semánticos que encontramos en foran. Así, como preposición, foran tiene sentidos metafóricos que no son heredados por la adposición compleja, cuando funciona también como preposición. En lo que se refiere a su función adverbial, el primitivo MOVIMIENTO, presente en el significado de foran 'hacia delante' no aparece en atforan, ya que éste se ha perdido al transferirse al compuesto del que forma parte. Por otra parte, como adverbio, atforan también muestra una reducción en el número de significados en comparación con foran. Mientras que foran tiene significados espaciales, temporales y otros de tipo metafórico, atforan, como adverbio, sólo mantiene un significado temporal 'de antemano'. 
11. Athindan: Como ya viene siendo una tendencia común con otras adposiciones complejas analizadas en este trabajo, el compuesto y el segundo integrante comparten el primitivo espacial que define uno de sus significados primarios: DETRÁs. También, como sucede recurrentemente con otros compuestos analizados aquí, athindan no incluye un significado de movimiento, que sí expresa su segundo componente hindan. Prestaré especial atención a cuestiones de este tipo porque podemos estar ante un principio de composición léxica en la formación de las adposiciones complejas. Hindan es una categoría polisémica y uno de sus sentidos, "desde atrás», tiene un significado dinámico que no aparece como parte del contenido semántico de athindan. Por tanto, hindan es exponente de MOVIMIENTO, primitivo que por el contrario no subyace al significado de athindan.

\section{Resultados Y Discusión}

El análisis del valor semántico de las adposiciones complejas en relación con sus elementos componentes permite extraer una serie de conclusiones que aclaran las cuestiones que han motivado este trabajo. Recordemos que las cuatro cuestiones planteadas en la Sección I responden a una cuestión heurística: la definición de los patrones combinatorios de los primitivos semánticos en el léxico espacial, particularmente en lo que afecta a patrones de composición semántica. Una vez explicados estos particulares, a la luz del análisis efectuado, se podrá dilucidar la evolución diacrónica de estos elementos hasta su situación actual y extraer las reglas de composición que dominan la formación de estas adposiciones.

\subsection{Contenido semántico de la adposición resultante en relación con los constituyentes}

En lo que respecta a este tema, tenemos que prestar atención a qué primitivos de los elementos componentes están todavía activos en el contenido semántico de la adposición compleja resultante. Además, tenemos que investigar si el compuesto es exponente de primitivos semánticos que no están presentes en ninguna de las adposiciones integrantes. En cuanto al proceso de composición que subyace a una adposición compleja, hay dos cuestiones básicas que tienen que ser consideradas: Una en conexión con el asunto de la compatibilidad semántica, a saber, las condiciones que vinculan a dos adposiciones específicamente y no a otras. La segunda cuestión que resta por tratar tiene que ver con las reglas sintácticas internas activas en estos 
elementos complejos que determinan un orden específico. En otras palabras, el factor que determina qué elemento controla la adposición compleja.

Un tercer problema que espera ser tratado es el de encontrar tendencias recurrentes en el contenido semántico de las adposiciones generadas. Cuando los predicados aparecen con argumentos, ven su significado especificado. Una situación similar puede identificarse aquí estimando que el segundo elemento, con una significación más concreta, funciona como un argumento. Esto explica que todos los compuestos hereden el significado del segundo elemento componente. Al mismo tiempo, el significado de éste se altera en diferentes formas cuando se integra en el compuesto. Tras estudiar la Tabla 3.1, hallamos que, cuando el primitivo semántico MOVIMIENTo está presente en una de las adposiciones controladas, no se mantiene en el compuesto. Por ejemplo, hindan y ufan expresan una relación dinámica entre el trayector y el hito, que no se mantiene en los compuestos donde se incorporan. Esto se puede atribuir al significado locativo estático dominante en on y at. Además, muy pocos compuestos ofrecen un significado que no se encuentre previamente presente en el componente controlado. Estos compuestos son onbutan y ongean. Este hecho justifica la desaparición de la gran parte de las adposiciones complejas. Otras, a pesar de haber sobrevivido hasta nuestros días, han caído totalmente en desuso como onforan 'afore', que se mantiene viva en expresiones cristalizadas como aforesaid o aforementioned 'anteriormente mencionado', relegadas al ámbito académico o formal. La creación de elementos compuestos cuyo significado a grandes rasgos es expresado por uno de sus componentes va en contra del principio de economía del lenguaje. Debería señalarse que en los casos en los que el significado de estas adposiciones complejas es diferente al de sus constituyentes, los primitivos que se utilizan para explicar este significado resultante sí están presentes, de todos modos, en los componentes.

\subsection{Las incompatibilidades semánticas que evita la combinación de algunas adposiciones}

La ecuación a resolver es si ciertas combinaciones podrían ser consideradas «imposibles» desde una perspectiva semántica. La Tabla 3.1 muestra que in, on y at comparten el primitivo semántico LUGAR/DONDE. A simple vista, puede parecer que estas adposiciones, siendo exponentes del mismo primitivo semántico, deberían poder combinarse con los mismos elementos, pero un examen más detallado hace que esta intuición no se sostenga. La polisemia está detrás de los distintos patrones combinatorios que encontramos. 
Con objeto de comprender la información incluida en la Tabla 3.1, es necesario algún conocimiento de inglés antiguo con respecto a las adposiciones in, on y at. Particularmente, en lo referido a su uso como preposiciones donde las diferencias afloran con mayor claridad. Los datos analizados aquí muestran que la preposición on es la que está involucrada en un número claramente más alto de adposiciones complejas. El punto crucial es que, como muestra en su función de preposición, on ostentaba en inglés antiguo una flexibilidad mayor que las otras dos preposiciones a la hora de expresar locación. En efecto, expresaba locación en casos en los que hoy en día esperaríamos encontrar in o at. Por ejemplo, se utilizaba para referirse a escenas espaciales que incluían continentes, mares, países, regiones, provincias, y otras divisiones geofísicas, tales como ciudades, etc. (Ludskær-Nielsen 1993). A consecuencia de eso, los primitivos CONTACTO y ARRIBA no eran tan representativos en el contenido léxico de esta adposición como lo son en inglés contemporáneo. El hecho de que en los textos donde estas preposiciones se alternan in perfilase un sentido de inclusión indica que el primitivo DENTRO ya era notorio en el uso de esta adposición en esa época y, por ende, estaba sujeto a reglas de distribución semántica distintivas. De hecho, in sólo se combina con la adposición on, menos específica, en estos procesos de composición. Estas combinaciones aparecen en las tablas anteriores en los compuestos innan y oninnan.

Finalmente, la preposición at en inglés antiguo, como en inglés moderno, se utilizaba cuando la locación era conceptualizada desde una perspectiva remota. Por otro lado, en escenas espaciales que comprendían objetos, y estaban impregnadas, por tanto, de un fuerte matiz funcional también encontrábamos esta preposición. No obstante, a diferencia del inglés actual, en el que la preposición at realiza esta labor prácticamente en exclusividad (p. ej., at the table 'en la mesa'), en inglés antiguo era a menudo la preposición on la que establecía este tipo de relación espacio-funcional.

Por esta razón, podemos concluir que las adposiciones que son altamente específicas, como muestran los primitivos que las ejemplifican, no son susceptibles de combinarse en posición de control con otras bases para constituir una unidad morfológica. Por ejemplo, no he encontrado en inglés antiguo adposiciones formadas por dos elementos proyectivos. Los elementos espaciales proyectivos como above 'arriba', expresan la perspectiva de los hablantes y otros factores deícticos (Herskovits 1986; Levinson 2003; Bierwisch 1967). Por esta razón, estos términos codifican relaciones espaciales que están prominentemente definidas en comparación con las relaciones expresadas por las preposiciones topológicas como in, on y at. Por consiguiente, el número de combinaciones que pueden ser consideradas como candidatas a producir compuestos se reduce notablemente. 


\subsection{La organización sintáctica interna que encontramos en estas adposiciones}

$\mathrm{Al}$ investigar una característica común en estos elementos que pueda ser evaluada como causa de la situación que acabamos de describir, el escenario es bastante claro: las adposiciones in, on y at son exponentes del mismo significado locativo espacial, LUGAR/DONDE. Este significado está menos elaborado desde un punto de vista configuracional que el de las adposiciones controladas por in, on y at. Esto se debe al hecho de que las preposiciones topológicas, por su adaptabilidad espacial, son susceptibles de aparecer en un número considerable de contextos. Esta adaptabilidad explica que en inglés antiguo fueran utilizadas en contextos similares, a diferencia de lo que sucede hoy en día. En inglés moderno esta adaptabilidad ha sido limitada porque la distribución de estas preposiciones está constreñida por la configuración geométrica de los hitos con los cuales se combinan.

En consecuencia, la vaguedad del primitivo LUGAR/DONDE que es ejemplificado por estas adposiciones, puede explicar la miríada de patrones combinatorios en los cuales toman parte. En este caso específico, controlan elementos que son exponentes de primitivos menos susceptibles de acomodarse a contingencias contextuales. Concretamente, entre los elementos controlados encontramos con cierta frecuencia adposiciones proyectivas. Según estos datos, deducimos que esta especificidad semántica no sólo bloquea la combinación de dos primitivos con significado espacial proyectivo, sino que establece su función u ordenación sintáctica dentro de la adposición compleja. Este hecho demuestra que la estructuración sintáctica de estos elementos está fuertemente determinada por aspectos semánticos, estudiados anteriormente.

Esta situación puede explicarse en términos de la organización predicado-argumento. Mientras el predicado presenta un contenido genérico fuera de contexto, su significado se concreta al combinarse con los correspondientes argumentos, que aparecen como elementos especificadores de su contenido. Una situación equiparable se da con in, on y at. Esta situación ratifica una premisa básica del MSN, la gramática no sólo es dependiente del significado sino que está fuertemente determinada por el mismo.

\subsection{Evolución diacrónica y principios de composición semántica de las adposiciones estudiadas}

Un dato que contribuye a explicar la desaparición de estas adposiciones complejas es que los elementos controlados ven su significado considerablemente simplificado a favor de significados puramente es- 
paciales. Por esa razón, pierden significados provenientes de extensiones metafóricas en el proceso de integración en el compuesto. Por ejemplo, onuppan no mantiene el significado temporal expresado por uppan. Esta situación es coherente con la pérdida de los significados dinámicos, como se indicó anteriormente, lo que apunta también a una simplificación del significado del elemento controlado. De esta manera, los hablantes podrían encontrar el elemento simple original mucho más útil. Factor éste que también menoscaba la economía del lenguaje.

En efecto, la evolución diacrónica experimentada por estas adposiciones complejas muestra una tendencia a caer en desuso cuando el elemento controlado o base, que proporciona al compuesto especificidad en su significado, pierde parte de su contenido semántico mientras que uno de sus significados obtiene más preponderancia. Esto aparece ilustrado en la Tabla 2.1 donde encontramos el caso de onuppan. Como cabía esperar, a la luz de estos hechos, las tres adposiciones complejas que han sobrevivido hasta el inglés actual comparten una característica: tienen un sentido que en ese momento no podía constatarse en ninguno de los elementos integrantes. Esta situación se da en las adposiciones onbutan (actual about 'sobre'), onforan (actual afore 'ante'), y ongean (actual against 'contra').

Es interesante notar que onbutan, ongean y onforan son exponentes de uno de los primitivos que caracteriza la adposición dominante o de control: CONTACTO. En cualquier caso, los contextos donde se usan contrastan con aquéllos donde la preposición on aparece, y esto es debido a la integración de los elementos controlados por on en el significado de la adposición resultante. Por lo tanto, según los datos analizados, las adposiciones complejas que tenían más posibilidades de sobrevivir presentan dos características relevantes. En primer lugar, son exponentes de uno de los primitivos semánticos ejemplificados por el elemento dominante de la adposición. En segundo lugar, el proceso de composición ha dado lugar a al menos un significado que constituye una innovación con respecto a los elementos integrantes.

A la luz de estos resultados, propongo un conjunto de Reglas Combinatorias que explican los patrones composicionales seguidos por los primitivos espaciales en estas adposiciones complejas:

Regla 1: El nivel de especificidad de los primitivos semánticos está restringido en sus posibilidades de combinación. Por lo tanto, no es viable que dos primitivos que definan relaciones espaciales con un alto grado de especificidad (por ejemplo, proyectivas) converjan en una nueva unidad léxica.

Regla 2: Los primitivos de movimiento desaparecen a favor de relaciones espaciales estáticas.

Regla 3: La integración no genera nuevos primitivos semánticos. 


\section{CONCLUSIÓN}

Este estudio ha ofrecido un análisis basado en el MSN que puede explicar cómo se combinan los primitivos semánticos de dicha metalengua composicionalmente para conformar la semántica compleja de ciertas adposiciones en inglés antiguo. Las ventajas de haber utilizado el MSN son diversas. En primer lugar, en línea con una de las premisas básicas de este modelo, demuestra que la gramática universal está basada en el significado. Concretamente, este análisis ha fundamentado la sintaxis interna de las adposiciones complejas en inglés antiguo en el significado de los elementos componentes. Los datos demuestran taxativamente que las adposiciones que aparecen controlando los compuestos muestran características semánticas específicas como un nivel de concreción menor en cuanto a la relación estructural que expresan. Según los resultados del examen, concluyo que no debe considerarse aleatoria la presencia recurrente de in, on y at en posición inicial frente la inexistencia de adposiciones proyectivas con una función predicativa dentro del compuesto. Por otro lado, los elementos con un significado más elaborado llevan a cabo el papel argumental con respecto a dichos predicados. Por lo tanto, este análisis refrenda la posición teórica de que las propiedades estructurales de una gramática se derivan de los significados articulados por dicha gramática.

En segundo lugar, en lo que se refiere a la implementación metodológica, los semanticistas somos conscientes de las complicaciones a las que nos enfrentamos en un análisis léxico. La dificultad aumenta al estudiar elementos resultantes de un proceso de composición, lo que nos obliga a desentrañar los condicionamientos del significado emergente así como la estructura que lo articula. Esta complicación aumenta cuando se trata de la unión de elementos de una misma clase léxica cerrada. Estos escollos se derivan, primero, de la aparente imposibilidad, en el caso particular de las adposiciones, de separar las adiciones semánticas procedentes del contexto de lo que pertenece al contenido propio del término. En segundo lugar, debo señalar la necesidad de distinguir entre polisemia de vaguedad semántica. De nuevo, una cuestión que ofrece especial dificultad en el caso de las adposiciones, cuyos referentes no son entidades específicas del mundo real sino que surgen de la percepción de una relación entre dos entidades físicas o abstractas. En este sentido, también puedo argumentar por qué el MSN constituye un marco conveniente para entender los procesos que subyacen a la composición de adposiciones. Se trata de un sistema integrado que durante treinta años ha demostrado su capacidad para describir con acierto cientos de análisis efectuados tanto con respecto al significado léxico, como gramatical y elocutivo. Además, los primitivos semánticos universales que propone abren una vía inteligible y testable para analizar la combina- 
ción de elementos altamente polisémicos porque contribuyen a superar las interferencias resultantes de interpretaciones contextuales, así como a reducir la miríada de sentidos que provienen tanto de la polisemia como de la vaguedad de un término. Esto se consigue gracias al carácter atómico y comprehensivo de estos universales que establecen relaciones unívocas con sus exponentes o en portmanteau.

Por lo expuesto en el párrafo anterior, se puede afirmar que el MSN contribuye a resolver los complejos problemas a los que se enfrenta un lingüista al estudiar la semántica de las adposiciones. Estos problemas se manifiestan en la mesnada de trabajos sobre preposiciones que han visto la luz en las últimas tres décadas. Estos trabajos no logran poner de acuerdo a los lingüistas que los producen, y desde mi punto de vista, la complejidad del objeto de estudio da lugar a ciertas construcciones formales de análisis establecidas ad hoc para satisfacer los objetivos de los autores. El ámbito de aplicación en el que han funcionado hasta ahora los primitivos semánticos es lo suficientemente amplio como para evitar precisamente esta rémora, que limita la precisión de un estudio semántico y pone en tela de juicio sus conclusiones. Por si fuera poco, el carácter pseudomatemático del modelo en el caso concreto de mi investigación me ha permitido extraer reglas que definen el comportamiento de las adposiciones complejas. El carácter transferible del modelo, que avala su universalidad, permite constatar las conclusiones obtenidas en este trabajo reduplicando la aplicación exacta que yo he efectuado del mismo a otros períodos de la lengua inglesa y a otras lenguas.

En cuanto a la contribución que este trabajo supone en el MSN, son dos las que podemos señalar. Por un lado, destaca las posibilidades de este modelo a la hora de realizar predicciones con respecto a la evolución de ciertos elementos léxicos que no están sujetos a restricciones referenciales como los sustantivos que aluden a objetos. Me refiero a que son elementos de la clase cerrada con un valor estructural, cuya evolución depende de percepciones y decisiones de los hablantes en cuanto a las relaciones que dichos elementos son capaces de establecer. En otras palabras, este trabajo no sólo refuerza la viabilidad sincrónica del modelo, sino también las posibilidades que presenta a nivel de análisis diacrónicos. Concretamente, los primitivos semánticos me han permitido delinear una explicación semántica que justifica la desaparición de unas adposiciones complejas y la pervivencia de otras, ofreciendo los resultados del análisis coherencia absoluta en estas dos facetas.

Sin embargo, mientras que lo anterior constituye lo que yo denominaría un resultado colateral del análisis, el objetivo central de este trabajo, tal y como se expone en la introducción, es demostrar que existen tendencias estables en el comportamiento combinatorio de estos elementos. La más relevante a mi juicio ha sido la constatación de que los elementos con un nivel alto de especificidad en su contenido semánti- 
co no confluyen en la formación de una adposición compuesta. Entre otras razones, se puede apuntar la incompatibilidad semántica y por tanto, la dificultad de seleccionar uno o más primitivos semánticos que conformen el elemento compuesto emergente. En lugar de eso, es más habitual la confluencia de adposiciones simples cuyos significados se complementan dentro de una estructura de predicado-argumento, lo que de nuevo nos conduce a propugnar, en consonancia con el MSN, la dependencia de la gramática con respecto al significado.

En cuanto a la mención de nuevas líneas de investigación que puedan surgir de este trabajo yo apuntaría hacia la realización de un trabajo similar con adposiciones complejas o locuciones adverbiales en otra lengua. De este trabajo se deriva, mediante la aplicación del método inductivo, una vía de descripción que demuestra que las reglas propuestas aquí se manifiestan en cualquier elemento léxico que haya requerido la composición de primitivos semánticos espaciales en su conformación.

Finalmente, me gustaría señalar que a pesar de la cantidad de trabajos que estudian el lenguaje espacial dentro de la escuela cognitivista, el comportamiento combinatorio del léxico espacial a nivel morfo-semántico no ha recibido suficiente atención hasta ahora. Por otro lado, deben enfatizarse las ventajas que un modelo de orientación estructuralista como el MSN presenta a la hora de implementarlo en el análisis de elementos con un grado de polisemia tan elevado como el de las adposiciones. De hecho, yo me permitiría hablar de un antecedente en el excelente trabajo de Bennet 1975. Encuentro interesantes paralelos entre los primitivos semánticos del MSN y el análisis composicional que este lingüista efectúa de las preposiciones inglesas. Pero, sobre todo, se pueden establecer analogías de gran interés heurístico entre los primitivos semánticos y el significado esquemático propuesto por Langacker 1987, el autor de la gramática cognitivista más influyente hasta la fecha.

Dicho de otro modo, ambas propuestas de análisis podrían ser objeto de combinación incluso en los sectores cognitivistas más radicales, puesto que, en mi opinión, los primitivos semánticos exhiben a nivel lingüístico muchas de las ventajas de ciertas construcciones cognitivistas de tipo analógico que han visto la luz bajo los auspicios de esta escuela. Pero, además, los primitivos están refrendados por un sistema altamente integrado y son aplicables, tanto en una relación unívoca como a través de portmanteaus, a elementos de otra naturaleza como verbos o sustantivos. Concretamente, su carácter proposicional permite llegar allí donde las representaciones diagramáticas pueden ser objeto de carencias. Se llega así a un punto vital dentro de los postulados cognitivos que consistiría en encontrar un modelo que englobe tanto elementos de representación analógicos como proposicionales, lo cual es coherente con el procesamiento de la información por parte de los hablantes, que recurre a ambos sistemas en la construcción de conceptos. 


\section{REFERENCIAS BIBLIOGRÁFICAS}

BennetT, D. C. (1975): Spatial and temporal uses of English prepositions, Londres, Longman.

BIERWISCH, M. (1967): «Some semantic universals of german adjectivals», Foundations of Language 3, pp. 1-36.

de la Cruz Cabanillas, I. (2006): «Semantic primes in Old English: a preliminary study of descriptors», 18th International SELIM Conference.

Clark Hall, John R. (1894/1960): A concise Anglo-Saxon dictionary, Cambridge, Cambridge University Press.

GODDARD, C. (2002): «On and on: verbal explications for a polysemic network». Cognitive Linguistics 13, 3, pp. 277-294.

-, y WierzBickA, A. (eds.) (2002): Meaning and universal grammar. Theory and empirical findings, Vol. I, Ámsterdam, John Benjamins.

GuARDDOn ANELo, M. C. (2006): «A cognitive approach to the diachronic study of prepositions», en Vázquez, Martínez, J. M. y Ron Vaz, P. (eds.), The historical linguistics - cognitive linguistics interface, Universidad de Huelva, pp. 147-166.

Herskovits, A. (1986): Language and spatial cognition. An interdisciplinary study of the prepositions in English, Cambridge, Cambridge University Press.

Johnson, M. (1987): The body in the mind. The bodily basis of meaning, imagination, and reason, Chicago y Londres, The University of Chicago Press.

Lakoff, G. y Johnson, M. (1980): Metaphors we live by, Chicago, The University of Chicago Press.

LANGACKER, R. (1987): Foundations of cognitive grammar: theoretical prerequisites, Vol. I, Stanford, Stanford University Press.

Levinson, S. C. (2003): Space in language and cognition, Cambridge, Cambridge University Press.

Lundsker-Nielsen, T. (1993): Prepositions in Old and Middle English. A study of prepositional syntax and the semantics of at, in and on in some Old and Middle English texts, Odense University Press.

MARTín ARISTA, J. (2005): «A preliminary analysis of old english semantic primes», 2005 Conference of ALS, held at Monash University.

-, (en prensa): Lexical negation in Old English.

-, (en prensa): «Unification and separation in a functional theory of morphology», en van Valin, R. (ed.), 2006 RRG Conference, Berlín, Mouton de Gruyter.

-, Caballero L.; González, E. e IbáÑez, A. (en prensa): Nerthus: an online lexical database of Old English.

—, y MARTÍN DE LA Rosa, V. (2006): «Old English semantic primes: substantives, determiners and quantifiers», Atlantis 28, 2, pp. 9-28.

TALMY, L. (1996): «Fictive motion in language and ception» en Bloom, P., Peterson, M., Nadel, L. y Garrett, M. (eds.), Language and space, Cambridge, MA, MIT Press, pp. 211-276.

WierzbickA, A. (1989): «Semantic primitives: the expanding set», Quaderni di Semantica 10, 2, pp. 309-332.

—, (1993): «Why do we say IN April, ON Thursday, AT 10 o'clock? In search of an explanation», Studies in Language 17, 2, pp. 437-454.

—, (2002): «Semantic primes and linguistic typology», en Goddard, C. y Wierzbicka, A. (eds.), Meaning and universal grammar. Theory and empirical findings, Vol. II, Ámsterdam, John Benjamins, pp. 65-144. 